\title{
Relación temporomandibulares con artritis reumatoidea
}

Lisboa CD, Pinochet CP, Larrucea CA. Relación de trastornos temporomandibulares con artritis reumatoidea. Rev Estomatol Herediana. 2007;17(1):16-21.

\section{RESUMEN}

Esta investigación determinó la frecuencia y características de los Trastornos Temporomandibulares (TTM) en personas que padecen Artritis Reumatoidea (AR), estableciendo relación entre la actividad de la enfermedad y su grado de incapacidad funcional. Se investigaron síntomas y signos de TTM en 71 pacientes (62 mujeres, 9 hombres) que cumplían con los criterios para AR según el Colegio Americano de Reumatología y que se encuentran en control en la Unidad de Reumatología del Hospital Regional de Talca. Determinados los síntomas y signos de TTM por examen clínico, fueron relacionados con los hallazgos clínicos y de laboratorio obtenidos de la evaluación reumatológica. El promedio de edad fue de 54,2 años, la duración de la enfermedad de 12,0 años, el $21,1 \%$ de los pacientes tenían activa la enfermedad, el $85,9 \%$ eran seropositivos para Factor Reumatoideo y el índice de incapacidad funcional de 2,02. Por otro lado los signos y síntomas de TTM mas destacados fueron el dolor a la palpación de la musculatura cervical $69 \%$, dolor a la palpación de la musculatura masticatoria $64,8 \%$, dolor a la palpación de la Articulación Temporomandibular (ATM) $69 \%$, ruidos en la ATM 60,6\%, rigidez matinal en la ATM 38\% y el 31\% de los pacientes presentó desviación en su trayectoria de apertura y cierre mandibular. Los pacientes con AR que se encontraban en periodos de actividad de la enfermedad o con curso clínico incapacitante, presentaron significativa mayor frecuencia de signos y síntomas asociados a TTM $(\mathrm{p}<0,05)$.

Palabras clave: ARTRITIS REUMATOIDE / TRASTORNOS DE LA ARTICULACIÓN TEMPOROMANDIBULAR.

Relashionship temporomandibular disorders and rheumatoid arthritis ABSTRACT

This investigation determined the frequency and characteristics of the Temporomandibular Disorders (TMD) in persons who suffer Rheumatoid Arthritis (RA), establishing relation between the activity of the disease and his degree of functional disability. Symptoms and signs were investigated in 71 patients (62 women, 9 men) that present the criteria for RA according to Rheumatology's American College and that were in control in Rheumatology's Unit of Talca's Regional Hospital. Once determined the symptoms and signs by clinical examination, they were related to the clinical finding and of laboratory obtained from the rheumatologic evaluation. The average age was 54.2 years, the duration was of the disease of 12.0 years, $21.1 \%$ of the patients had the disease active, $85.9 \%$ was seropositive for Rheumatoid Factor and the index of functional disability of 2.02 . On the other hand the more out-standing TMD signs and symptoms were the pain at the palpation of the cervical musculature $69 \%$, pain to the palpation of the masticatory musculature $64.8 \%$, pain to the palpation of the Temporomandibular Joint (TMJ) $69 \%$, noises in the ATM $60.6 \%$, morning rigidity in the ATM $38 \%$ and a $31 \%$ of the patients presented desviation in the opening and closing jaw trayectorie. The patients with AR who were in periods of activity of the disease or with incapacitating clinical course, presented a major significant frequency of signs and symptoms associated with TTM $(\mathrm{p}<0.05)$.

Key words: RHEUMATOID ARTHRITIS / TEMPOROMANDIBULAR JOINT DISORDERS

\section{Introducción}

El porcentaje del compromiso de la Articulación Temporomandibular (ATM) en la Artritis Reumatoidea (AR) resulta controversial. Algunos autores reportan un 20\%, mientras que otros comunican rangos que van desde el 40\% al 80\% dependiendo del grupo estudiado (1). Se estima en un $1 \%$ la prevalencia a nivel mundial (2,3). Las mujeres se afectan con una frecuencia de $3: 1$ en relación a los hombres (2).

La artritis reumatoidea (AR) es una enfermedad crónica que causa discapacidad, dos son las evaluaciones que se realizan con este fin, los marcadores de actividad inflamatoria de la AR, la velocidad de eritrosedimentación (VHS) y el daño articular; en contraste, las mediciones del desenlace son las que evalúan las consecuencias de la AR, es decir, las pérdidas que deja la enfermedad en discapacidad funcional y calidad de vida (4). Uno de estos indicadores corresponde al Índice de Incapacidad Funcional, obtenido mediante la aplicación de un cuestionario que contiene ocho preguntas básicas. De esta forma se obtienen valores que van desde 1,00 que indica que no hay disfunción, hasta 4,00 , que va indicando una disfunción progresiva (5).

En relación a los Trastornos
Cristian D. Lisboa ${ }^{1}$

Carmen P. Pinochet ${ }^{2}$

Carlos A. Larrucea ${ }^{3}$

${ }^{1}$ Cirujano Dentista.

${ }^{2}$ Medico Reumatólogo. Hospital Regional de Talca,

${ }^{3}$ Profesor Asistente. Facultad de Ciencias de la Salud.

Universidad de Talca.

\section{Correspondencia}

Carlos Alberto Larrucea Verdugo

Universidad de Talca

Av. Lircay s/n

Escuela de Odontología

Fono: 56-71-201503

e-mail: larrucea@utalca.cl

Recibido : 06 de agosto del 2007

Aceptado : 05 de octubre del 2007
Temporomandibulares (TTM), es un término que agrupa un número de problemas clínicos que involucran las ATM, musculatura masticatoria, o ambas, son un grupo de trastornos relacionados con el sistema masticatorio que tienen muchas características en común (6-7).

El compromiso de la AR en la ATM clínicamente produce un dolor sordo en la región preauricular durante la función, dolor a la palpación, rigidez matutina, chasquidos, crepitación y disminución de la fuerza de masticación (1). Otros síntomas presentes a menudo son sensibilidad e hinchazón sobre el área de la ATM. Los síntomas generalmen- 
te son de naturaleza transitoria, y sólo un pequeño porcentaje de pacientes con artritis de las ATM experimentará una incapacidad significativa en forma permanente. Los más consistentes hallazgos clínicos incluyen dolor a la palpación de las articulaciones y limitación en la apertura. La crepitación también puede ser evidente (8). Funcionalmente, el movimiento de la articulación como el que se realiza en la masticación o al hablar, causa dolor y puede estar limitado por la rigidez (9).

En cuanto a la Epidemiología, los trastornos de la ATM afectan con mayor frecuencia al sexo femenino con una relación 4: 1 y de 2:1 según otros autores. La franja etaria predominante es entre los 20 y 40 años; aunque otras referencias señalan que la mayor incidencia de afectación se encuentra entre los 21 a 30 años (10).

Un estudio de prevalencia de TTM en la ciudad de Talca, tomó una muestra al azar de 70 individuos (42 mujeres y 28 hombres), cuyas edades fluctuaron entre 21 y 50 años, encontrándose que el $92,8 \%$ de la población estudiada presentaba al menos un síntoma o signo relacionado con un TTM (11).

El síntoma que se presenta con mayor frecuencia es dolor, usualmente localizado en la ATM y/o en los músculos masticatorios. La masticación, fonación y otras funciones mandibulares generalmente agravan estos síntomas. Otras complicaciones incluyen ruidos articulares (clic, pop, chasquidos y crepitación) y limitación de los movimientos mandibulares (apertura y lateralidades). También pueden estar presentes síntomas auxiliares no específicos tales como cefaleas, dolor de cuello y otalgias (7).

El dolor orofacial es una queja común en las personas que padecen TTM, siendo frecuentemente relatados el dolor de los músculos masticatorios o de la ATM. La estimación del dolor es un paso muy importante en la evaluación inicial así como en los procesos siguientes para determinar la eficacia de las estrategias de manejo propuestas (12).

Los sonidos de la ATM son asociados con el diagnóstico de TTM, clasificados como clicks, crépito intenso, o crépito suave $(13,6)$.

El propósito de este estudio fue determinar los síntomas y signos relacionados con TTM en personas que padecen AR de acuerdo a su grado de severidad.

\section{Material y métodos}

Se tomó una muestra consecutiva de 71 pacientes adultos (62 mujeres, 9 hombres) con AR según los Criterios del Colegio Americano de Reumatología (ACR) (14), que acuden a control en la Unidad de Reumatología del Hospital Regional de Talca. Según criterios de exclusión se eliminaron de la muestra a aquellos pacientes que presentaban una o más de las siguientes condiciones: signos evidentes de Bruxismo; antecedentes de TTM actual o previo y un tiempo de evolución de la AR menor a 6 meses, desde el diagnóstico de la enfermedad.

Estos pacientes fueron evaluados, previo consentimiento informado, a través de dos exámenes, reumatológico y odontológico, ambos consecutivos en el mismo día, los resultados fueron consignados en una ficha clínica por separado para su posterior confrontación y análisis.

La evaluación de las articulaciones periféricas, distintas de la ATM y la actividad de la enfermedad fue realizada por la reumatóloga tratan- te. Se evaluó un total de 36 articulaciones diartrodiales: de la $2^{\circ}$ a la $5^{\circ}$ articulaciones metacarpofalángicas, de la $2^{\circ}$ a la $5^{\circ}$ articulaciones interfalángicas proximales, articulación interfalángica del pulgar, muñeca, codo, rodilla, tobillo y de la $1^{\circ}$ a la $5^{\circ}$ articulaciones metatarsofalángicas, todas bilaterales.

Esta evaluación se llevó a cabo sobre la base de un examen clínico y el análisis de resultados de los exámenes de laboratorio; ambos procedimientos comprendidos dentro del esquema habitual de seguimiento clínico y de laboratorio de la enfermedad, la duración de la AR, definida como el tiempo transcurrido desde el inicio de la enfermedad con sus síntomas y signos; número de articulaciones dolorosas; número de articulaciones inflamadas; factor reumatoideo (FR) y velocidad de hemosedimentación (VHS).

La actividad de la Artritis Reumatoidea fue definida por la presencia de al menos dos de los siguientes tres criterios (4):

- 6 ó más articulaciones dolorosas

- 3 ó más articulaciones inflamadas

- VHS de al menos $28 \mathrm{~mm} / \mathrm{h}$.

Con respecto al Grado de Incapacidad Funcional, éste fue evaluado a través del Índice de Incapacidad Funcional HAQ (Health Assessment Questionaire) (4-15).

La evaluación de los síntomas y signos fue llevada a cabo el mismo día de la evaluación reumatológica, por un operador capacitado en el Centro de Diagnóstico y Tratamiento de TTM de la Escuela de Odontología de la U. de Talca:

Síntomas: Dolor articular, Cefaleas, Dolor cervical, Rigidez matinal de ATM, Dificultades para mover la mandíbula. La intensidad del dolor fue evaluada a través de una escala visual análoga.

Signos: 
- Ruidos articulares: Clic (apertura, cierre, recíproco), Crepitación (leve, intensa)

- Dolor a la palpación de: Músculo Temporal, Masetero, Pterigoideo externo (examen funcional), Esternocleidomastoideo, Musculatura posterior del cuello.

- Alteraciones del desplazamiento mandibular: Limitación en la apertura o hipometría (menor a $35 \mathrm{~mm}$, distancia interincisal compensada al over-bite), limitación en lateralidad derecha (menor a $7 \mathrm{~mm}$ desde la línea media), limitación en lateralidad izquierda (menor a 7 mm desde la línea media), desviación y deflexión.

Todo el análisis estadístico fue realizado utilizando el programa SPSS 8.0 para Windows. Para establecer las correlaciones entre las distintas variables se realizaron las pruebas de chi cuadrado (prueba exacta de Fisher s y chi cuadrado de Pearson). La significancia estadística fue definida como p 0,05.

\section{Resultados}

El promedio de edad de los pacientes examinados fue de $54,24+12,5$ años (rango de 21-79 años) y su procedencia fue predominantemente urbana (56,3\%). El promedio de duración de la AR fue de 12,05+12,17

Tabla 1. Características clínicas de los pacientes.

\begin{tabular}{|c|c|}
\hline Descripción & valor \\
\hline $\begin{array}{l}\text { Promedio Índice de Incapacidad Funcional (HAQ) } \\
\text { (HA) }\end{array}$ & 2,02 \\
\hline No. de articulaciones periféricas dolorosas $\pm \mathrm{DE}$ & $0,86 \pm 1,9$ \\
\hline No. de articulaciones periféricas inflamadas $\pm \mathrm{DE}$ & $2,45+3,04$ \\
\hline VHS mm/h $\pm \mathrm{DE}$ & $29,27 \pm 17,4$ \\
\hline Hto $\pm \mathrm{DE}$ & $38,93+2,99$ \\
\hline Recuento plaquetario x103 /mm3 + DE & $295,7 \pm 84,3$ \\
\hline Factor Reumatoideo Positivo & $61(85,9 \%)$ \\
\hline AINEs & $62(87,3 \%)$ \\
\hline Prednisona < de 10 mg/día & $33(46,5 \%)$ \\
\hline Prednisona > de 10 mg/día & $28(39,4 \%)$ \\
\hline Metotrexato & $62(87,3 \%)$ \\
\hline Hidroxicloroquina & $2(2,8 \%)$ \\
\hline Azatioprina & $2(2,8 \%)$ \\
\hline
\end{tabular}

años y 61 pacientes $(85,9 \%)$ eran seropositivos para el FR. Al momento del examen, 15 pacientes (21,1\%) tenían activa su enfermedad. El Índice de incapacidad funcional alcanzó un valor promedio de 2,02. Las características clínicas de los pacientes con AR se muestran en la tabla 1.

La sensación de rigidez matinal de la ATM fue el síntoma específico relatado con mayor frecuencia por los pacientes (38,0\%), seguida por la dificultad para realizar movimientos mandibulares (19,7\%). Por otra parte, la cefalea fue el síntoma auxiliar o inespecífico reportado con mayor frecuencia, alcanzando un 35,2\% (Tabla 2).
Con respecto a los signos de TTM, el dolor a la palpación de la ATM y la musculatura cervical fueron los más prevalentes (ambos con 69,0\%). El dolor a la palpación de los músculos masticatorios se halló en $64,8 \%$ de los casos. La crepitación, leve e intensa, fue el ruido articular pesquisado con mayor frecuencia (36,7\%), seguido por el clic articular con un $23,9 \%$. No hubo una diferencia significativa entre los lados derecho e izquierdo al momento de la evaluación (Tabla 3).

Con respecto a las alteraciones en los desplazamientos mandibulares, la limitación en los movimientos de lateralidad fue el signo hallado con mayor frecuencia $(71,8 \%)$. Le

Tabla 2. Síntomas de trastornos temporomandibulares.

\begin{tabular}{lrc}
\hline Síntomas de TTM & $\mathrm{n}$ & $(\%)$ \\
\hline Específicos & & \\
Rigidez matinal de ATM & 27,00 & $(38,0)$ \\
$\quad$ Dificultad en movimientos mandibulares & 14,00 & $(19,7)$ \\
Dolor de ATM & 12,00 & $(16,9)$ \\
Intensidad Promedio (EVA) & 3,70 & \\
No Específicos & & \\
$\quad$ Cefaleas & 25,00 & $(35,2)$ \\
Intensidad Promedio (EVA) & 4,02 & \\
$\quad$ Dolor Cervical & 7,00 & $(9,9)$ \\
$\quad$ Intensidad Promedio (EVA) & 5,20 & \\
\hline EVA: Escala visual análoga & &
\end{tabular}

siguió, en segundo lugar, la desviación durante la apertura bucal (31,0\%) (Tabla 4).

$\mathrm{Al}$ analizar la correlación entre las distintas variables, se observó que los pacientes con AR activa y con mayor grado de incapacidad funcional presentaron con mayor frecuencia dolor a la palpación de sus ATM. Este resultado fue estadísticamente significativo $(\mathrm{p}<0,05)$.

No se encontró asociación entre el principal síntoma relatado por los pacientes (sensación de rigidez matinal de ATM) y los Índices reumatológicos. (Tablas 5 y 6 ) 
Tabla 3. Signos de trastornos temporomandibulares.

\begin{tabular}{|c|c|c|c|c|}
\hline Signo TTM & & $\begin{array}{l}\text { LD } \\
\mathrm{n}(\%)\end{array}$ & $\begin{array}{l}\text { LI } \\
\text { n (\%) }\end{array}$ & $\mathrm{n}(\%)$ \\
\hline \multicolumn{5}{|l|}{ Dolor a la palpación } \\
\hline ATM Lateral & & $35(49,3)$ & $28(39,4)$ & \\
\hline ATM Posterior & & $21(29,6)$ & $16(22,5)$ & \\
\hline Al menos una ATM & & & & $49(69,0)$ \\
\hline Intensidad Promedio (EVA) & 2,4 & & & \\
\hline Temporal anterior & & $7(9,9)$ & $5(7,0)$ & \\
\hline Temporal medio & & $17(23,9)$ & $15(21,1)$ & \\
\hline Temporal posterior & & $16(22,5)$ & $12(16,9)$ & \\
\hline Masetero superficial & & $24(33,8)$ & $27(38,0)$ & \\
\hline Masetero profundo & & $14(19,7)$ & $15(21,1)$ & \\
\hline Pterigoideo externo & & $4(5,6)$ & $6(8,5)$ & \\
\hline Al menos un músculo masticador & & & & $46(64,8)$ \\
\hline Intensidad Promedio (EVA) & 2,6 & & & \\
\hline ECM & & $28(39,4)$ & $26(36,6)$ & \\
\hline Posteriores del cuello & & $26(36,6)$ & $31(43,7)$ & \\
\hline Al menos un músculo cervical & & & & $49(69,0)$ \\
\hline Intensidad Promedio (EVA) & 3,4 & & & \\
\hline \multicolumn{5}{|l|}{ Auscultación ATM } \\
\hline Crepitación intensa & & $7(9,9)$ & $4(5,6)$ & \\
\hline Crepitación leve & & $14(19,7)$ & $18(25,4)$ & \\
\hline Crepitación en al menos una ATM & & & & $26(36,7)$ \\
\hline Clic en apertura & & $2(2,8)$ & $4(5,6)$ & \\
\hline Clic en cierre & & $7(9,9)$ & $3(4,2)$ & \\
\hline Clic recíproco & & $4(5,6)$ & $3(4,2)$ & \\
\hline Clic en al menos una ATM & & & & $17(23,9)$ \\
\hline
\end{tabular}

lores también fueron inferiores a los encontrados por Larrucea (11) $44,2 \%$ hecho atribuible al tratamiento farmacológico en los pacientes con AR de este estudio.

El signo de TTM más prevalente encontrado en este estudio, fue el dolor a la palpación de la ATM $(69 \%)$, resultado muy superior al $20 \%$ reportado por Kononen et al. (16) y similar al porcentaje reportado por Gynther et al. (50\%) (17), este último utilizó una muestra inferior a la nuestra, de 22 individuos, en una muestra equivalente Larrucea (11) encontró un $37,1 \%$ valor que indica claramente el grado de afectación de la ATM por la AR.

El dolor a la palpación de los músculos masticatorios se encontró con una frecuencia de $64,8 \%$, valor similar al reportado por Ettala-Ylitalo et al. $(53,3 \%)(18)$ y muy superior al encontrado por Laurell et al. (38\%) (19), en su estudio, Laurell examinó 204 pacientes suecos con

\section{LD: lado derecho, LI: lado izquierdo}

\section{Discusión}

Los principales síntomas de TTM hallados en este grupo de estudio fueron la rigidez matinal de la ATM $(38,0 \%)$ y la dificultad para realizar movimientos mandibulares $(19,7 \%)$. No se encontraron estudios previos donde hallan sido evaluados estos síntomas en pacientes con $\mathrm{AR}$, sin embargo antecedentes reportados por Larrucea et al., (11) en un grupo similar sin AR, se encontró un $31,4 \%$ de rigidez matinal y un $12,8 \%$ de dificultad para realizar movimientos mandibulares, lo que indicaría que la AR estaría actuando como un factor que incrementaría la prevalencia de estos síntomas.

El síntoma auxiliar o inespecífico mas frecuente, fue la cefalea $(35,2 \%)$, este valor es inferior al hallado por Koh et al. (7) (55\%), en
Tabla 4. Alteraciones del desplazamiento mandibular.

\begin{tabular}{lrc}
\hline Movimiento anormal & $\mathrm{n}$ & $(\%)$ \\
\hline Desviación & $22(31,0)$ \\
Deflexión & $3 \quad(4,2)$ \\
Limitación de apertura $(<35 \mathrm{~mm}$ interincisal) & $5(7,0)$ \\
Limitación lateralidad izquierda $(<7 \mathrm{~mm})$ & $51(71,8)$ \\
Limitación lateralidad derecha $(<7 \mathrm{~mm})$ & $51(71,8)$ \\
\hline
\end{tabular}

Tabla 5. Asociación entre dolor a la palpación, rigidez matinal de ATM e índices reumatológicos.

\begin{tabular}{lll}
\hline Índices reumatológicos & $\begin{array}{l}\text { dolor a la } \\
\text { palpación de ATM }\end{array}$ & $\begin{array}{l}\text { rigidez } \\
\text { matinal de ATM }\end{array}$ \\
\hline Actividad de la AR & $0,018 \mathrm{~S}$ & $0,543 \mathrm{NS}$ \\
Índice de incapacidad funcional & $0,048 \mathrm{~S}$ & $0,413 \mathrm{NS}$ \\
Factor reumatoideo & $0,628 \mathrm{NS}$ & $0,576 \mathrm{NS}$ \\
\hline
\end{tabular}

S: Significativo, NS: No significativo

este estudio, los autores plantean como posible causa del alto porcentaje, la presencia concomitante de TTM en seis pacientes, así como también la subluxación atloaxial en dos pacientes; los mencionados va-

AR, de los cuales sólo el $17 \%$ eran desdentados (todos portadores de prótesis), respecto al dolor a la palpación de los músculos masticatorios Larrucea (11) determinó un $37,1 \%$, la alta frecuencia de este signo en 
los pacientes con AR podría explicarse por el hecho conocido de la interrelación de la unidad cráneocérvico-facial, por lo que el deterioro de uno o mas de los constituyentes afecta al o los otros.

Con respecto a la crepitación de las ATM, en nuestro estudio ésta se presentó en $36,7 \%$ de los casos, este hallazgo es similar a los valores reportados por Laurell (30\%) (19) y Koh (32\%) (7), y difiere mucho de los valores reportados por otros autores, tales como: Larheim (16,6\%) (20), Gynther (63,6\%) (17) y Kononen (82\%) (16), creemos que esta diferencia puede deberse a la diferencia en el tamaño muestral; Larheim y Gynther trabajaron con 49 y 22 individuos respectivamente, por su parte, Kononen atribuye el alto porcentaje obtenido en su estudio a que la mayoría de los pacientes que conformaron su grupo de estudio (61 individuos) se encontraban en etapas tardías de la enfermedad, con todo el daño acumulado que esto implica.

La limitación de la apertura bucal tuvo una muy baja frecuencia en nuestro estudio (7\%), en investigaciones previas Ettala-Ylitalo et al. (18) reportaron un 31,7\%, mientras que Yoshida et al. (21) encontraron un porcentaje similar al nuestro (12,8\%). En el primer caso se examinó a 60 pacientes, mientras que Yoshida examinó a 218 personas. Resulta difícil explicar la diferencia entre los resultados de ambos estudios, creemos que la diferencia étnica entre las distintas poblaciones estudiadas (Finlandesa y Japonesa, respectivamente) podrían explicar en cierta forma esta situación, ya que antecedentes aportados por Larrucea (11) en pacientes sin AR encontró un $12,8 \%$, avalando el hecho que la limitación de la apertura es un signo que se relaciona con trastornos agudos mas que con TTM de tipo crónico.

$\mathrm{Al}$ realizar el análisis de correla- ción entre las distintas variables, se observó que los pacientes que presentaban una AR activa y con un mayor grado de incapacidad funcional presentaron con mayor frecuencia dolor a la palpación de sus ATM, esta diferencia fue estadísticamente significativa $(p<0,05)$, por lo tanto, podemos señalar que en pacientes con un mayor compromiso inflamatorio o con una AR más severa o incapacitante, deberíamos pensar inmediatamente en la posibilidad de encontrar sintomatología dolorosa a nivel de la ATM, a esto debemos agregar que se observó también una fuerte asociación entre el dolor a la palpación de las ATM y el uso de Metotrexato (dato no mostrado) en el tratamiento de los pacientes $(p=0,001)$, este fármaco fue utilizado en casos de AR mas severas, en conclusión, esta asociación ratifica lo observado en relación con el grado de incapacidad de los pacientes y su asociación con el dolor a la palpación de la ATM.

También se correlacionó (dato no mostrado) el principal síntoma obtenido de nuestro estudio (sensación de rigidez matinal de la ATM) con el principal signo observado (dolor a la palpación de las ATM), de esto se puede concluir, que las personas que relataron sensación de rigidez matinal de ATM presentaron en forma significativa una mayor frecuencia de dolor a la palpación de sus articulaciones $(p=0,004)$.

Los principales síntomas de TTM relatados por el grupo de pacientes en estudio fueron Rigidez matinal de ATM y Cefaleas a repetición, no se encontró asociación estadísticamente significativa entre éstos síntomas y los Índices Reumatológicos, sin embargo es interesante destacar que estos síntomas no son los más prevalentes en la población $\sin \mathrm{AR}$, siendo el dolor de cuello, musculatura masticatoria y saltos articulares los más importantes en este grupo (11), hecho atribuible a la mayor actividad de los pacientes sanos respecto a los enfermos.

Los principales signos de TTM obtenidos en este estudio fueron dolor a la palpación de la ATM y dolor a la palpación de la musculatura cervical. El dolor a la palpación de la ATM se relacionó significativamente $(\mathrm{p}<0,05)$ con la actividad de las AR y el grado de incapacidad funcional de los pacientes en estudio. La asociación no fue positiva al momento de relacionar el dolor a la palpación de la ATM con la presencia del FR a nivel sanguíneo, de igual modo es interesante destacar que estos signos no son los más prevalentes en la población sin AR, siendo la alteración de la dinámica articular y los ruidos articulares los más importantes en este grupo (11), hecho que podría sugerir que la enfermedad estaría produciendo alteraciones tendientes a provocar síntomas de TTM como la rigidez matinal de la ATM y la dificultad para realizar movimientos mandibulares, y signos como el dolor a la palpación de la ATM y el dolor a la palpación de los músculos masticatorios en estos pacientes.

\section{Referencias bibliográficas}

1. Bascones A, Manso FJ. Dolor Orofacial, diagnóstico y tratamiento. 1ra ed. Madrid: Ediciones Avances; 1997.

2. Massardo L. Apuntes en reumatología: Artritis reumatoidea. Disponible en: http:/ /escuela.med.puc.cl/publ/ Apuntes Reumatologia/ ArtritisReumatoidea.html

3. Abello M. Epidemiologia e impacto de la artritis reumatoide. Revista Colombiana de Reumatología. 2000; 7(1). Disponible en: http:// encolombia.com/reuma722000epidemiologia.htm

4. González H. Instrumentos de 
medición en artritis reumatoide. Revista Colombiana de Reumatología. 2000; 7(1). Disponible en: http:// encolombia.com/reuma722000instrumentos.htm

5. Pincus T, Callahan LF, Brooks RH, Fuchs HA, Olsen NJ, Kaye JJ. Self-report questionnaire scores in rheumatoid arthritis compared with traditional physical, radiographic, and laboratory measures. Ann Intern Med. 1989; 110(4):259-66.

6. Patología de ATM. Definición. Odontocat. El portal de la odontología. Disponible en: http:// w w w . od on tocat . com/ patoatm 2.htm

7. Koh ET, Yap AU, Koh CK, Chee TS, Chan SP, Boudville IC. Temporomandibular disorders in rheumatoid arthritis. J Rheumatol. 1999; 26(9):1918-22.

8. Lynch MA, Greenberg M. Burket's oral medicine: diagnosis and treatment. 9th. Ed. Philadepphia: J. B. Lippincott Co.; 1994.

9. Shafer WG, et al. Tratado de patología bucal. 4a ed. México DF: Interamericana; 1987.

10. Aguilar M. Trastornos de la articulación temporomandibular. Centro de Información en otorrinolaringología y fonoaudiología. La Revista Digital. 2001; 1(1). Disponible en: http:// w w w . c i o f. co m.ar/ residentes.htm.

11. Larrucea C, Castro R, Vera A. Estudio de prevalencia de signos y síntomas de trastornos temporomandibulares (TTM) en un grupo de adultos de Talca, Chile. Rev Dent Chile. 2002; 93(3):28-33.

12. Conti PC, de Azevedo LR, de Souza NV, Ferreira FV. Pain measurement in TMD patients: evaluation of precision and sensitivity of different scales. J Oral Rehabil. 2001; 28(6):534-9.

13.Leader JK, Robert Boston J, Rudy TE, Greco CM, Zaki HS, Henteleff HB. Quantitative description of temporomandibular joint sounds: defining clicking, popping, egg shell crackling and footsteps on gravel. J Oral Rehabil. 2001; 28(5):466-78.

14. West Sterling G. Secretos de la reumatología. México DF: McGraw-Hill Interamericana; 1997.

15.Klippel JH, Dieppe PA. Rheumatology. 2nd ed. London: Mosby; 1998.

16.Kononen M, Wenneberg B, Kallenberg A. Craniomandibular disorders in rheumatoid arthritis, psoriatic arthritis, and ankylosing spondylitis. A clinical study. Acta Odontol Scand. 1992; 50(5):2817.

17. Gynther GW, Holmlund AB, Reinholt FP, Lindblad S.
Temporomandibular joint involvement in generalized osteoarthritis and rheumatoid arthritis: a clinical, arthroscopic, histologic, and immunohistochemical study. Int J Oral Maxillofac Surg. 1997; 26(1):106.

18. Ettala-Ylitalo UM, Syrjanen S, Halonen P. Functional disturbances of the masticatory system related to temporomandibular joint involvement by rheumatoid arthritis. J Oral Rehabil. 1987; 14(5):415-27.

19. Laurell L, Hugoson A, Hakansson J, Pettersson B, Sjostrom L, Berglof FE, Berglof $\mathrm{K}$. General oral status in adults with rheumatoid arthritis. Community Dent Oral Epidemiol. 1989; 17(5):230-3 .

20.Larheim TA, Storhaug K, Tveito L. Temporomandibular joint involvement and dental occlusion in a group of adults with rheumatoid arthritis. Acta Odontol Scand. 1983; 41(5):3019.

21. Yoshida A, Higuchi Y, Kondo M, Tabata O, Ohishi M. Range of motion of the temporomandibular joint in rheumatoid arthritis: relationship to the severity of disease. Cranio. 1998; 16(3):1627. 